\title{
Vapor pressure and liquid density of fluorinated alcohols: experimental, simulation and GC-SAFT-VR predictions
}

\author{
Gonçalo M. C. Silva ${ }^{1}$, Pedro Morgado ${ }^{1}$, Jessica D. Haley, ${ }^{1}$ Víctor Manuel Trejos Montoya, ${ }^{1}$ \\ Clare McCabe ${ }^{2,3}$, Luís F. G. Martins ${ }^{4}$ and Eduardo J. M. Filipe ${ }^{1 *}$ \\ ${ }^{1}$ Centro de Química Estrutural, Instituto Superior Técnico, Universidade de Lisboa, 1049-001 \\ Lisboa, Portugal \\ ${ }^{2}$ Department of Chemical and Biomolecular Engineering and ${ }^{3}$ Department of Chemistry, \\ Vanderbilt University, Nashville TN 37235, U.S.A. \\ ${ }^{4}$ Centro de Química de Évora, Universidade de Évora, Rua Romão Ramalho, 59, 7000-671 \\ Évora, Portugal
}

\begin{abstract}
The vapor pressure of four liquid 1H,1H-perfluoroalcohols $\left(\mathrm{CF}_{3}\left(\mathrm{CF}_{2}\right)_{\mathrm{n}}\left(\mathrm{CH}_{2}\right) \mathrm{OH}, \mathrm{n}=1,2,3\right.$, 4), often called odd-fluorotelomer alcohols, was measured as a function of temperature between $278 \mathrm{~K}$ and $328 \mathrm{~K}$. Liquid densities were also measured for a temperature range between $278 \mathrm{~K}$ and $353 \mathrm{~K}$. Molar enthalpies of vaporization were calculated from the experimental data. The results are compared with data from the literature for other perfluoroalcohols as well as with the equivalent hydrogenated alcohols. The results were modeled and interpreted using molecular dynamics simulations and the GC-SAFT-VR equation of state.
\end{abstract}

\section{Introduction}

Highly fluorinated compounds have become important substances, both from an industrial and fundamental point of view. Fluorinated alcohols in particular, find application in numerous commercial products from textile protection agents and fire-fighting foams, to detergents, paints, and as precursors in the production of fluorinated polymers [1].

Fluorotelomer alcohols are linear highly fluorinated molecules possessing a terminal $\mathrm{OH}$ group, with the general formula, $\mathrm{CF}_{3}\left(\mathrm{CF}_{2}\right)_{\mathrm{n}}\left(\mathrm{CH}_{2}\right)_{\mathrm{m}} \mathrm{OH}$ that is often summarized as $\mathrm{n}+1: \mathrm{m}$ FTOH. This molecular structure results in an enhanced amphiphilic character compared to hydrogenated alcohols, as fluorinated chains are known to be more hydrophobic than their hydrogenated analogues. Fluorotelomers can be divided into two major groups: odd FTOH with $\mathrm{m}=1$ and even FTOH when $\mathrm{m}=2$. In an effort to understand and model these molecules, experimental properties such as vapor pressure and densities were measured and enthalpies of vaporization calculated.

This work is part of a project in which experimental measurements, molecular simulation techniques and theoretical calculations have been simultaneously used to elucidate the properties of fluorinated substances and their mixtures. Using this approach, we have recently 
reported diffusion coefficients of fluorinated alcohols in aqueous solutions [2]. The behavior of mixtures of fluorinated and hydrogenated alcohols has also been studied [3]. These mixtures display a very complex behaviour when compared with mixtures of hydrogenated alcohols and mixtures of alkanes and perfluoroalkanes. The excess volumes are large and positive (unlike those of mixtures of hydrogenated alcohols [4]) while the excess enthalpies are large and negative (contrasting with those of mixtures of alkanes and perfluoroalkanes [5]). This peculiar behaviour results from a delicate balance between the weak dispersion forces between the hydrogenated and fluorinated groups and a preferential hydrogen bond between the hydrogenated and the fluorinated alcohols.

Following this line of work, we now present new experimental data for the vapor pressures and liquid densities of four liquid $1 \mathrm{H}, 1 \mathrm{H}$-perfluoroalcohols $\left(\mathrm{CF}_{3}\left(\mathrm{CF}_{2}\right)_{n} \mathrm{CH}_{2} \mathrm{OH}, \mathrm{n}=1,2,3,4\right)$ as a function of temperature. Molar enthalpies of vaporization were calculated from the experimental data and the results compared with data from the literature for other perfluoroalcohols as well as their equivalent hydrogenated alcohols. Vapor pressure measurements were previously reported for the longer chained $\left(\mathrm{CF}_{3}\left(\mathrm{CF}_{2}\right)_{n} \mathrm{CH}_{2} \mathrm{OH}, \mathrm{n}=5-9\right)$ odd fluorotelomers [6], but for the short chains studied in this work, experimental data has not been reported or is of insufficient accuracy. Molecular dynamics simulations were also performed and provide molecular level insight into the experimental results. Experimental molar enthalpies of vaporization and densities were used to validate the force field used in the molecular dynamics simulations. Additionally, the GC-SAFT-VR equation was used to predict the experimental results. Excellent agreement has been found between the theoretical predictions and the experimental results.

\section{Experimental and simulation techniques}

\subsection{Purification and characterization}

1H,1H-perfluoropropan-1-ol (2:1 FTOH, CAS number:422-05-9), 1H,1H-perfluorobutan-1ol (3:1 FTOH, CAS number:375-01-9), 1H,1H-perfluoropentan-1-ol (4:1 FTOH, CAS number:355-28-2) and 1H,1H-perfluorohexan-1-ol (5:1 FTOH, CAS number:423-46-1) were purchased from Apollo Scientific Ltd; a 98\% purity was indicated for all the alcohols except perfluoropentanol, for which a $97 \%$ purity was claimed. Prior to their use, the compounds were dried with VWR Prolabo 4A molecular sieves to a maximum water content of $500 \mathrm{ppm}$ (analysed by Karl-Fischer coulometry) and their purity was confirmed by ${ }^{1} \mathrm{H}$ NMR spectroscopy.

Table 1 - Sample Provenance and purity for 2:1, 3:1, 4:1 and 5:1 FTOH.

\begin{tabular}{|c|c|c|c|c|c|}
\hline Compound & Source & $\begin{array}{l}\text { Claimed } \\
\text { Purity }\end{array}$ & $\begin{array}{l}\text { Purification } \\
\text { Method }\end{array}$ & $\begin{array}{l}\text { Analysis } \\
\text { Methods }\end{array}$ & $\begin{array}{c}\text { Water } \\
\text { Content }\end{array}$ \\
\hline $\begin{array}{c}2,2,3,3,3 \text {-Pentafluoro-1-propanol } \\
(2: 1 \text { FTOH })\end{array}$ & $\begin{array}{c}\text { Apollo } \\
\text { Scientific Ltd. }\end{array}$ & 0.98 & $\begin{array}{c}\text { Drying in } \\
\text { Molecular Sieves }\end{array}$ & - & - \\
\hline $\begin{array}{l}\text { 2,2,3,3,4,4,4-Heptafluoro-1- } \\
\text { butanol }(3: 1 \text { FTOH) }\end{array}$ & $\begin{array}{c}\text { Apollo } \\
\text { Scientific Ltd. }\end{array}$ & 0.98 & $\begin{array}{c}\text { Drying in } \\
\text { Molecular Sieves }\end{array}$ & $\begin{array}{c}\text { KF } \\
\text { Coulometry }\end{array}$ & $472 \mathrm{ppm}$ \\
\hline $\begin{array}{c}\text { 2,2,3,3,4,4,5,5,5-Nonafluoro-1- } \\
\text { pentanol (4:1 FTOH) }\end{array}$ & $\begin{array}{c}\text { Apollo } \\
\text { Scientific Ltd. }\end{array}$ & 0.97 & $\begin{array}{l}\text { Drying in } \\
\text { Molecular Sieves }\end{array}$ & - & - \\
\hline
\end{tabular}


2,2,3,3,4,4,5,5,6,6,6-Undecafluoro1-hexanol (5:1 FTOH)
Apollo

Scientific Ltd.
0.98
$\mathrm{KF}$
Coulometry $316 \mathrm{ppm}$

\subsection{Vapor pressure measurements}

The vapor pressure of the fluoroalcohols was measured in the 278 to $328 \mathrm{~K}$ temperature range, with the exception of the $3: 1 \mathrm{FTOH}$ that was measured from 298 to $328 \mathrm{~K}$. The measurements were made using a static apparatus previously described [7], which consists of a spherical glass cell connected to a vacuum line and to a pressure transducer. The cell is immersed in a thermostatic water bath controlled by a Hart Scientific 2100 PID temperature controller. The temperature of the liquid was measured using a calibrated Pt100 temperature sensor connected to a Keithley $20006 \frac{1}{2}$ digital multimeter, with an absolute uncertainty of $0.05 \mathrm{~K}$. The temperature stability and uniformity during a measurement is estimated to be better than $0.01 \mathrm{~K}$. The pressure was measured with a Paroscientific Series 1000 quartz absolute pressure transducer connected to a Paroscientific model 715 display unit. The pressure sensor is capable of measuring up to $100 \mathrm{psia}(0.69 \mathrm{MPa})$, with a resolution of $0.0001 \%$ of the full scale and has an integrated temperature compensation system. While measuring, the connecting line between the glass cell and the pressure transducer was kept at a higher temperature than the bath's in order to avoid condensation of the vapor.

The liquids were submitted to freezing in liquid nitrogen, vacuum pumping and melting cycles to degas them. The samples were further purged to the vacuum line for a few seconds while agitating the liquid. The latter procedure was repeated until the measured vapor pressure was reproducible, confirming that no other volatile species were present. The temperature was then changed and the pressure recorded after stabilization. Measurements were made in paths of increasing and decreasing temperature, in order to reduce the possibilities of systematic error.

\subsection{Density measurements}

The liquid densities of the fluoroalcohols were measured in an Anton Paar DMA 5000 vibrating-tube densimeter. The instrument was calibrated with water (distilled, purified with a Mili-Q 185 plus water purification system, and freshly boiled) and air at $20.000{ }^{\circ} \mathrm{C}$, taking into account atmospheric pressure. The calibration was checked with water over the whole range of operating temperatures, and the maximum deviation from literature values was found to be less than $0.00002 \mathrm{~g} . \mathrm{cm}^{-3}$. The density of air was verified at the beginning of each series of measurements to ensure the cleanliness of the measurement cell.

\subsection{Molecular dynamics simulations}

Molecular dynamics simulations were carried out to obtain molecular-level information on the behaviour of the studied systems, using an all atom force field based on OPLS all atom force field (OPLS-AA) [8]. For the perfluoroalkyl segments of the molecules, the OPLS-AA parameters from Watkins and Jorgensen [9] were used, while the $-\mathrm{CF}_{2} \mathrm{CH}_{2} \mathrm{OH}$ segment was modeled with the parameters developed by Duffy for trifluoroethanol [10,11], adjusting the partial charge of the fluorinated carbon to maintain the neutrality of the molecule. The 
missing dihedral torsion parameters, for the fluorinated-hydrogenated junction, were taken from the work of Pádua [12]. The simulations were performed using the DL_POLY [13] simulation engine, in the $N p T$ ensemble at $1 \mathrm{~atm}$ and $298.15 \mathrm{~K}$. All bonds involving hydrogen atoms were constrained to their equilibrium lengths, using the SHAKE algorithm. Pressure and temperature were kept constant using the Nosé-Hoover barostat and thermostat, with relaxation times of 2 and $0.5 \mathrm{ps}$, respectively. A total of $20 \mathrm{~ns}$ were simulated with a $2 \mathrm{fs}$ time step for the liquid phases. In the gas phases, for each compound, 20 independent simulations of a single molecule system, started from different initial configurations, were performed for 20 ns each with a 1 fs time step. A cut-off of $14 \AA$ was used for the Lennard-Jones and Coulomb potentials, with analytic tail corrections applied for the former and the Ewald sum method incorporated to calculate the long-range interactions for the latter.

The system densities were obtained directly from the average values of the system volume in the $N p T$ simulations and the enthalpies of vaporization calculated using equation 1

$$
\Delta H_{\text {vap.calcd }}=E_{\text {config, },}-E_{\text {config }, l}+R T
$$

where $E_{\text {config,g }}$ and $E_{\text {config,l }}$ are the configurational molar energies of the liquid and gas phases and $R T$ corresponds to the PV-work term for an ideal gas. The work term for the liquid phase is considered negligible. Gas and liquid phase simulations were performed to obtain the configurational energies.

The average number of hydrogen bonds in the liquid fluorotelomer alcohols was obtained by integration of the oxygen - hydroxyl hydrogen radial distribution functions (RDF). Hydrogen bonding was defined by considering all intermolecular $\mathrm{O}-\mathrm{H}$ pairs with a separation smaller than $2.67 \AA$ (the first minimum of the RDF) [3]. The analysis of the molecular conformations was performed using the TRAVIS software [14].

\section{GC-SAFT-VR}

The GC-SAFT-VR equation combines the SAFT-VR [15] equation with a group contribution (GC) [16] approach that allows for the description of chains built up from segments of different size and/or energy of interaction $[17,18]$. The model allows for the location of the functional groups and association sites within a molecule to be specified, enabling the heterogeneity in molecular structure to be captured within a SAFT model.

In the GC-SAFT-VR approach, molecules are described by tangentially bonded segments in which each type of segment represents a functional group present in the molecule $[16,19]$. The segments representing each functional group interact via a square well potential, which can be described by,

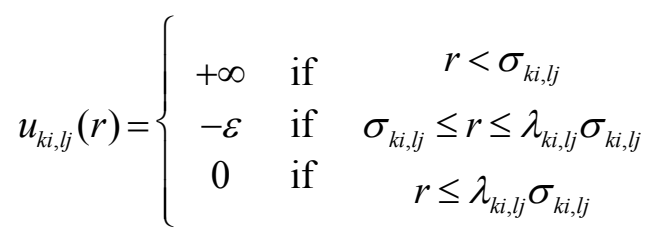

where $u_{k i, l j}$ represents the interaction between a functional group of type $i$ present in molecule $k$ with a functional group of type $j$ in molecule $l, \sigma$ is the segment diameter, $\varepsilon$ is the depth of the square well, $\lambda$ is the potential range, and $r$ is the distance between the two functional 
groups. The unlike size and energy interactions can be obtained from the Lorentz-Berthelot combining rules expressed by,

$$
\begin{gathered}
\sigma_{i j}=\frac{\sigma_{i i}+\sigma_{j j}}{2} \\
\varepsilon_{i j}=\sqrt{\varepsilon_{i i} \varepsilon_{i j}}
\end{gathered}
$$

and the unlike potential range is given by,

$$
\lambda_{i j}=\frac{\lambda_{i i} \sigma_{i i}+\lambda_{j j} \sigma_{j j}}{\sigma_{i i}+\sigma_{i j}}
$$

The GC-SAFT-VR equation of state is written in terms of the total Helmholtz free energy, expressed as a sum of four separate contributions:

$$
\frac{A}{N k_{B} T}=\frac{A^{\text {ideal }}}{N k_{B} T}+\frac{A^{\text {mono }}}{N k_{B} T}+\frac{A^{\text {chain }}}{N k_{B} T}+\frac{A^{\text {assoc }}}{N k_{B} T}
$$

where $N$ is the total number of molecules in the system, $T$ is the temperature, and $k_{B}$ is the Boltzmann constant. In equation $6, A^{\text {ideal }}$ is the ideal free energy, $A^{\text {mono }}$ is the contribution to the free energy due to the monomer segments, $A^{\text {chain }}$ is the contribution due to the formation of bonds between monomer segments, and $A^{a s s o c}$ is the contribution due to association. Since the theory has been presented previously [16,18] only a brief overview of the main expressions are provided below.

The ideal Helmholtz free energy, $A^{\text {ideal }}$, is given by,

$$
\frac{A^{\text {ideal }}}{N k_{B} T}=\sum_{i=1}^{n_{\text {components }}} x_{i} \ln \left(\rho_{i} \Lambda_{i}^{3}\right)-1
$$

where $n_{\text {components }}$ represents the number of pure components, $\rho_{i}=N_{i} / V$ (the molecular number density of chains of component $i$ ), $x_{i}$ is the mole fractions of component $i$ in the mixture, $A_{i}$ is the thermal de Broglie wavelength, $N$ is the total number of molecules in the system, $T$ is the temperature, and $k_{B}$ is the Boltzmann constant.

The monomer free energy, $A^{\text {mono }}$, is given by a second order high temperature expansion using Barker and Henderson perturbation theory for mixtures [20],

$$
\frac{A^{\text {mono }}}{N k T}=\sum_{i=1}^{n_{\text {componens }}} \sum_{k=1}^{n_{k}^{\prime}} m_{k i} x_{i}\left(a^{H S}+\frac{a_{1}}{k_{B} T}+\frac{a_{2}}{\left(k_{B} T\right)^{2}}\right)
$$

where $a^{H S}$ is the hard sphere reference term and $a_{1}$ and $a_{2}$ are the first and second order perturbation terms; the first sum is over the components in the system, the second sum is over all $n_{k}$ types of functional groups within each component and $m_{k i}$ is the number of segments of type $k$ in chains of component $i$.

The chain term, $A^{\text {chain }}$, for a mixture of heterosegmented chain molecules is given by,

$$
\frac{A^{\text {chain }}}{N k T}=-\sum_{i=1}^{n} x_{i} \sum_{k j} \ln y_{i k, i j}^{S W}\left(\sigma_{i k, i j}\right)
$$


where the first sum is over all components in the mixture and the second sum considers the chain formation and connectivity of the segments within a given chain. The background correlation function is given by,

$$
y_{k i, k j}^{S W}\left(\sigma_{k i, k j}\right)=\exp \left(\frac{-\varepsilon_{k i, k j}}{k_{B} T}\right) g_{k i, k j}^{S W}\left(\sigma_{k i, k j}\right)
$$

where $g_{k i, k j}^{S W}\left(\sigma_{k i, k j}\right)$ is the radial distribution function for the square well monomers at the contact distance $\sigma_{k i, k j}$ and is approximated by a first-order high-temperature perturbation expansion.

Finally, the contribution due to association, $\left(A^{A s s o c}\right)[15]$, interactions between sites on different functional groups that form the molecules of interest is expressed as:

$\frac{A^{A s s o c}}{N k_{B} T}=\sum_{i=1}^{n} x_{i} \sum_{k=1}^{n_{k}^{\prime}} v_{i k} \sum_{a=1}^{n s_{i}^{\prime}} n_{k a}\left(\ln X_{i k a}+\frac{1-X_{i k a}}{2}\right)$

where the first sum is over the number of components $n$, the second one is over all types of functional groups in the molecule $k$ and the third is over the total number of site types. The fraction of molecules of type $i$ not bonded at site $a, \mathrm{X}_{i k a}$, is obtained from the solution of the mass balance equations and is given in terms of the total number density for the system.

In this work, the FTOH molecules studied have been modelled using the GC-SAFT-VR equation in a totally predictive approach. The fluoroalcohols, with formula $\mathrm{CF}_{3}\left(\mathrm{CF}_{2}\right)_{\mathrm{n}} \mathrm{CH}_{2} \mathrm{OH}$, are comprised of the $\mathrm{CF}_{3}, \mathrm{CF}_{2}, \mathrm{CH}_{2}$, and terminal $\mathrm{OH}$ groups. The parameters for which (m, $\varepsilon, \sigma$, and $\lambda$, and the association parameters for the $\mathrm{OH}$ group), were all taken from previous work with the GC-SAFT-VR equation [16,21,22]. In the GC-SAFTVR approach the model parameters are determined by fitting to experimental vapor pressure and saturated liquid density data for molecules containing the groups of interest. Specifically, the alkanes were used to determine $\mathrm{CH}_{2}$ parameters, perfluoroalkanes and perfluoroalkylalkanes were used to determine the $\mathrm{CF}_{3}$ and $\mathrm{CF}_{2}$ parameters, and the $n$-alcohols between butanol and decanol were used to determine the parameters for the terminal $\mathrm{OH}$ group. At this point, it is important to note that in the usual homonuclear model of SAFT the contribution to the free energy due to bonding at separate sites are independent, and as such, the location of sites on a molecule is arbitrary; the sites are in actuality averaged over the whole molecule. However, in the GC-SAFT-VR equation, because of the hetero-segmented chain model used, the location of the association sites can be specified on a given functional group and hence their position within the model chain defined [15]. Utilizing this approach, the GC-SAFT-VR theory is used to successfully predict, without any reliance on experimental data, the thermodynamic properties of the fluoroalcohol molecules.

\section{Results and discussion}

\subsection{Vapor pressures and enthalpies of vaporization}

The experimental vapor pressures as a function of temperature of the studied FTOH are reported in table 2 and plotted in Figure 1. 
Table 2. Experimental vapor pressures for 2:1, 3:1, 4:1 and 5:1 FTOH. ${ }^{\mathrm{a}}$

\begin{tabular}{|c|c|c|c|c|c|c|c|c|c|c|c|}
\hline \multicolumn{3}{|c|}{ 2:1 FTOH } & \multicolumn{3}{|c|}{ 3:1 FTOH } & \multicolumn{3}{|c|}{ 4:1 FTOH } & \multicolumn{3}{|c|}{ 5:1 FTOH } \\
\hline $\mathbf{T} / \mathbf{K}$ & $\begin{array}{c}\mathbf{P}_{\text {vap }} / \\
\mathbf{k P a}\end{array}$ & $\begin{array}{l}U(p) / \\
\mathbf{k P a}\end{array}$ & $\mathbf{T} / \mathbf{K}$ & $\begin{array}{l}\mathbf{P}_{\text {vap }} / \\
\mathbf{k P a}\end{array}$ & $\begin{array}{l}U(p) / \\
\mathbf{k P a}\end{array}$ & $\mathbf{T} / \mathbf{K}$ & $\begin{array}{c}\mathbf{P}_{\text {vap }} / \\
\mathbf{k P a}\end{array}$ & $\begin{array}{l}U(p) / \\
\mathbf{k P a}\end{array}$ & $\mathbf{T} / \mathbf{K}$ & $\begin{array}{c}\mathbf{P}_{\text {vap }} / \\
\mathbf{k P a}\end{array}$ & $\begin{array}{l}U(p) / \\
\mathbf{k P a}\end{array}$ \\
\hline 298.08 & 6.2055 & 0.024 & 278.14 & 0.7213 & 0.008 & 278.14 & 0.2236 & 0.006 & 278.14 & 0.0688 & 0.005 \\
\hline 303.05 & 8.4158 & 0.030 & 283.10 & 1.0470 & 0.009 & 283.10 & 0.3363 & 0.006 & 283.10 & 0.1098 & 0.005 \\
\hline 308.04 & 11.3058 & 0.037 & 288.08 & 1.4954 & 0.010 & 288.07 & 0.5020 & 0.007 & 288.08 & 0.1712 & 0.006 \\
\hline 313.01 & 14.9656 & 0.046 & 293.08 & 2.1173 & 0.012 & 293.06 & 0.7329 & 0.008 & 293.08 & 0.2615 & 0.006 \\
\hline 317.98 & 19.5864 & 0.057 & 298.10 & 2.9653 & 0.015 & 298.09 & 1.0700 & 0.009 & 298.13 & 0.3944 & 0.007 \\
\hline 322.96 & 25.4116 & 0.071 & 303.07 & 4.0862 & 0.018 & 303.10 & 1.5312 & 0.010 & 303.10 & 0.5819 & 0.007 \\
\hline 327.93 & 32.4857 & 0.088 & 308.04 & 5.5515 & 0.022 & 308.06 & 2.1523 & 0.012 & 308.06 & 0.8405 & 0.008 \\
\hline & & & 313.03 & 7.4728 & 0.027 & 313.02 & 2.9794 & 0.015 & 313.01 & 1.2052 & 0.009 \\
\hline & & & 317.98 & 9.9047 & 0.034 & 317.98 & 4.0707 & 0.018 & 317.98 & 1.7082 & 0.011 \\
\hline & & & 322.95 & 13.1446 & 0.042 & 322.99 & 5.4963 & 0.022 & 322.93 & 2.3494 & 0.013 \\
\hline & & & 327.91 & 17.2579 & 0.052 & 327.95 & 7.3149 & 0.027 & 327.93 & 3.2499 & 0.016 \\
\hline
\end{tabular}

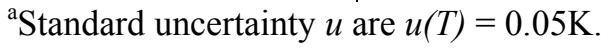

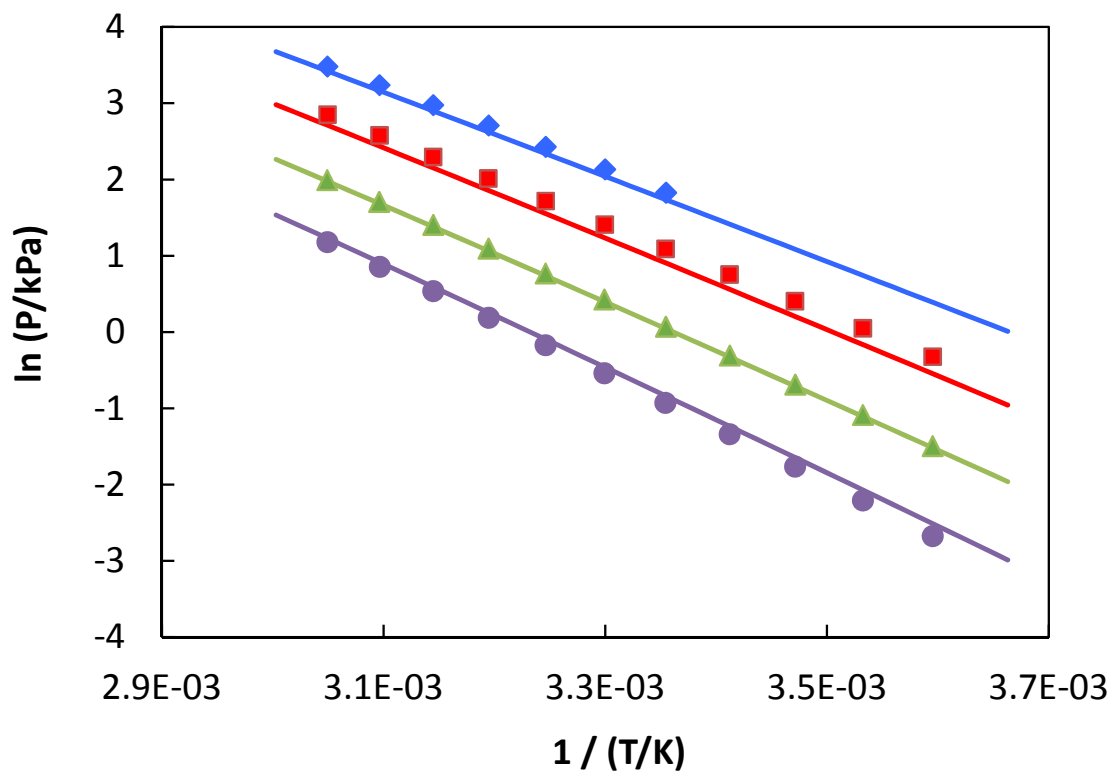

Figure 1. Experimental vapor pressures for 2:1 $(\bullet), 3: 1(\boldsymbol{\bullet}), 4: 1(\boldsymbol{\Delta})$ and 5:1 $\mathrm{FTOH}(\bullet)$ and GC-SAFT-VR predictions (lines).

The experimental results were correlated with the Antoine equation

$$
\ln (p / k P a)=A-\frac{B}{(T / K)+C}
$$


where $p$ is the vapor pressure, $T$ is the temperature and $A, B$ and $C$ are the adjustable Antoine coefficients. The obtained coefficients reproduce the vapor pressure data within the experimental uncertainty and are presented in table 3, along with the root mean square deviation (RMSD) of the fit and the average percent deviation, which is defined as:

$$
\Delta p / p \%=\frac{100}{n} \sum\left|\frac{p_{\exp }-p_{c a l}}{p_{\exp }}\right|
$$

where $n$ is the number of experimental points.

Table 3. Antoine equation coefficients

\begin{tabular}{|c|c|c|c|c|c|}
\hline Compound & A & B & C & RMSD / kPa & $\Delta \boldsymbol{p} / \boldsymbol{p}(\mathbf{\%})$ \\
\hline 2:1 FTOH & 15.439 & 2931.877 & -82.748 & 0.015 & 0.1 \\
\hline 3:1 FTOH & 18.821 & 4808.731 & -26.973 & 0.035 & 0.2 \\
\hline 4:1 FTOH & 16.278 & 3608.616 & -75.398 & 0.004 & 0.5 \\
\hline 5:1 FTOH & 25.162 & 8615.885 & 31.895 & 0.042 & 1.7 \\
\hline
\end{tabular}

The measured vapor pressures for 2:1 FTOH and 3:1 FTOH are compared with data from the literature in figure 2. In this work we have measured the vapor pressure of 2:1 FTOH between $298 \mathrm{~K}$ and $328 \mathrm{~K}$, complementing the data of Meeks and Goldfarb [23] at a lower temperature range, between $273 \mathrm{~K}$ and $298 \mathrm{~K}$. As can be seen, both data sets compare favourably. For 3:1 FTOH, however, the vapor pressure measured in this work is much lower than the previously reported values, also by Meeks and Goldfarb [23]. The relative differences can be as high as $100 \%$, which is obviously much higher than the experimental uncertainties. The temperature dependence is also very different for both data sets and, as will be shown, the earlier data [23] is not consistent with those observed for the other substances in the FTOH family. It is thus very unlikely that such a discrepancy is due to experimental error and suggests the existence of a volatile impurity in the sample of Meeks and Goldfarb [23]. 


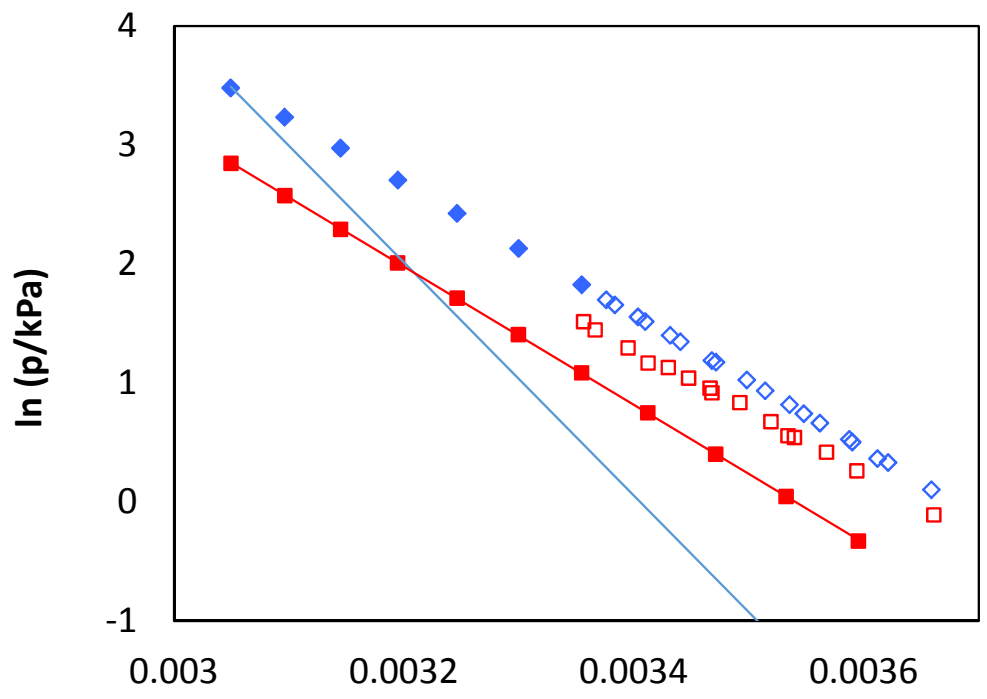

$1 /(\mathrm{T} / \mathrm{K})$

Figure 2. Logarithmic representation of the vapor pressures with $1 / \mathrm{T}$ for: $2: 1 \mathrm{FTOH}$, this work $(\diamond)$ and Meeks and Goldfarb [23] $(\diamond)$; 3:1 FTOH, this work (ם), Meeks and Goldfarb $[23](\square)$. The lines correspond to linear (Clausius-Clapeyron) fits to the experimental results.

The GC-SAFT-VR predictions for the vapor pressure of the fluoroalcohols are compared with the experimental data in figure 1. As can be seen, the theory is able to reproduce the vapor pressure in close agreement with the experimental results. It should be emphasized that the theoretical results are pure predictions, as none of the model parameters was fitted to the experimental data.

The enthalpies of vaporization of the studied FTOH were estimated from the vapor pressure data using the Clausius-Clapeyron equation, assuming that the enthalpy of vaporization is constant in the measured temperature range and that the vapor phase behaves as an ideal gas. This should be a reasonable approximation since the measured pressures are very low. The calculated enthalpies of vaporization are reported in table 4 and plotted in figure 3 . Also shown are the enthalpies of vaporization from the literature [6,23] for 1:1 FTOH, 2:1 FTOH, 3:1 FTOH and for longer chain alcohols from 6:1 FTOH to 10:1 FTOH. As can be seen, the vaporization enthalpy for the FTOH family increases almost linearly with chain length, displaying an average increment of $4.8 \mathrm{~kJ} \mathrm{~mol}^{-1}$ per each additional $\mathrm{CF}_{2}$ group. Regarding the data of Meeks and Goldfarb [23], the vaporization enthalpy obtained for $2: 1 \mathrm{FTOH}$ is $4 \%$ higher than the value now reported, whereas the vaporization enthalpy of $3: 1 \mathrm{FTOH}$ is $11 \%$ lower, deviating visibly from the trend established by the other data points. We interpret this deviation as a further confirmation that the literature data for 3:1 FTOH is inaccurate, probably due to the presence of a volatile impurity. 
Table 4. Molar enthalpies of vaporization of 1H,1H-perfluoroalcohols.

\begin{tabular}{|c|c|c|c|c|c|}
\hline Compound & $\begin{array}{l}\Delta \mathbf{H}_{\text {vap,exp }}{ }^{\mathrm{a}} \\
/ \mathrm{kJ} \mathrm{mol}^{-1} \\
\end{array}$ & $\begin{array}{c}\boldsymbol{u}\left(\Delta \mathrm{H}_{\mathrm{vap}, \mathrm{exp}}\right) \\
/ \mathrm{kJ} \mathrm{mol}^{-1} \\
\end{array}$ & $\begin{array}{c}\Delta \mathbf{H}_{\text {vap,sim }} \\
/ \mathrm{kJ} \mathrm{mol}^{-1}\end{array}$ & $\begin{array}{c}\boldsymbol{u}\left(\Delta \mathbf{H}_{\mathrm{vap}, \mathrm{sim}}\right) \\
/ \mathrm{kJ} \mathrm{mol}^{-1} \\
\end{array}$ & $\begin{array}{c}\Delta \mathbf{H}_{\text {vap,lit }} \\
/ / \mathbf{k J ~ m o l}^{-1} \\
\end{array}$ \\
\hline $1: 1 \mathrm{FTOH}$ & - & - & - & - & $44.5^{\mathrm{b}}$ \\
\hline 2:1 FTOH & 45.1 & 0.2 & 47.1 & 1.2 & $46.9^{\mathrm{b}}$ \\
\hline 3:1 FTOH & 48.3 & 0.1 & 53.2 & 1.0 & $43.5^{\mathrm{b}}$ \\
\hline 4:1 FTOH & 53.2 & 0.2 & 59.0 & 0.8 & - \\
\hline 5:1 FTOH & 58.6 & 0.3 & 61.9 & 0.8 & - \\
\hline 6:1 FTOH & - & - & - & - & $64.6^{\mathrm{c}}$ \\
\hline 7:1 FTOH & - & - & - & - & $68.4^{\mathrm{c}}$ \\
\hline 8:1 FTOH & - & - & - & - & $72.4^{\mathrm{c}}$ \\
\hline 9:1 FTOH & - & - & - & - & $80.5^{\mathrm{c}}$ \\
\hline 10:1 FTOH & - & - & - & - & $84.4^{\mathrm{c}}$ \\
\hline
\end{tabular}
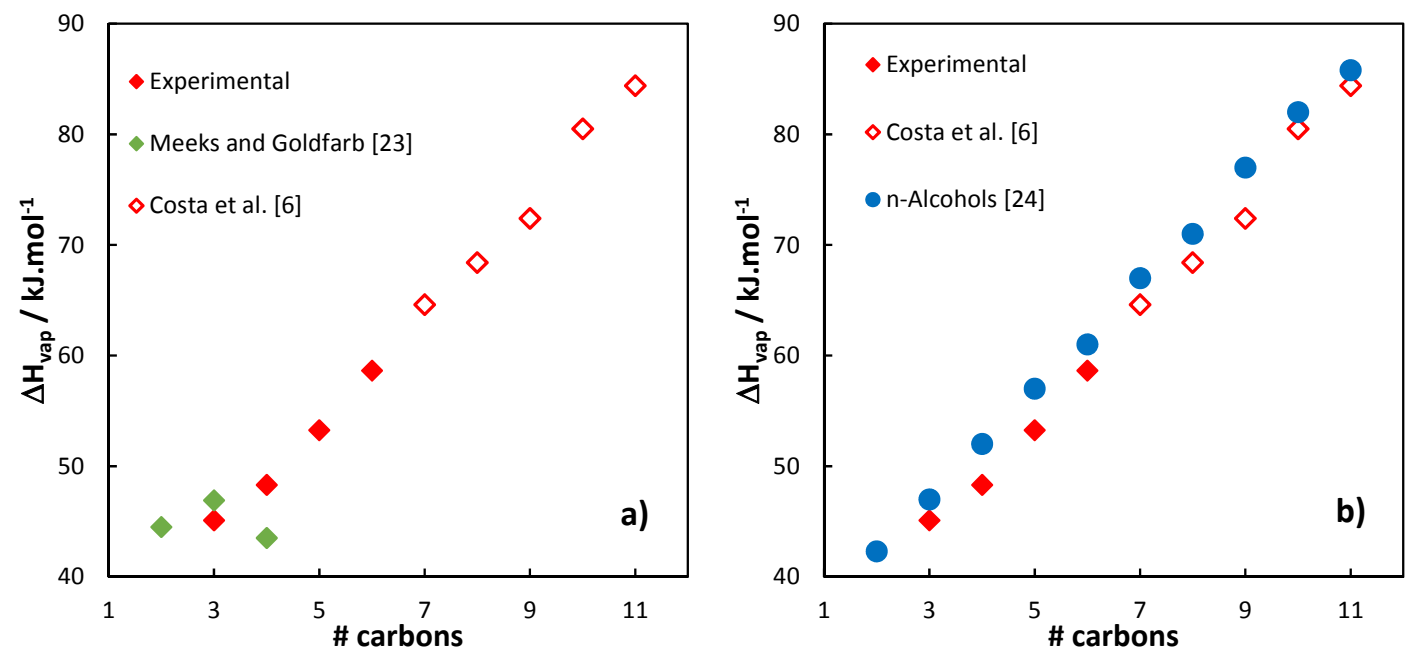

Figure 3. Molar enthalpies of vaporization of the FTOH family as a function of chain length. a) comparison with literature results; b) comparison with the hydrogenated alcohols analogues.

In figure $3 b$, it can be seen that the enthalpies of vaporization of FTOH are consistently lower by $2-4 \mathrm{~kJ} / \mathrm{mol}$, than those of the hydrogenated alcohols [24], which also present a linear trend with approximately the same slope. In a recent paper [6], Costa et al. concluded that the higher volatility of the longer fluorinated alcohols relative to their hydrogenated analogues is entropically driven, since they found that the enthalpies of vaporization were very similar for both families while the corresponding entropies were slightly higher. The results for the shorter FTOH obtained in the present work, however, confirm that the difference in vaporization enthalpies is larger than the experimental uncertainty, indicating that enthalpic contributions to the higher volatility of FTOH may be significant and cannot be ignored. 
The low values of the $\Delta \mathrm{H}_{\mathrm{vap}}$ of $\mathrm{FTOH}$, especially when taking into account their high molecular weights, may result from several factors including the low cohesiveness of perfluorinated liquids. However, they are also consistent with a lesser degree of hydrogen bonding when compared to hydrogenated alcohols, which would reduce the cohesiveness of the liquid phase. Additionally, intramolecular gauche interactions between the positively charged hydroxyl $\mathrm{H}$ and the electronegative fluorine atoms may stabilize these molecules in the gas phase [25], also contributing to a smaller enthalpy of vaporization. As will be discussed below, the molecular dynamics results support both hypotheses.

Estimations of the enthalpies of vaporization were also obtained from the molecular simulations as described in section 2.4 above. The results are reported in table 4 and compared with the experimental results in figure 4. As can be seen, the simulations overestimate the vaporization enthalpies by 4 to $11 \%$.

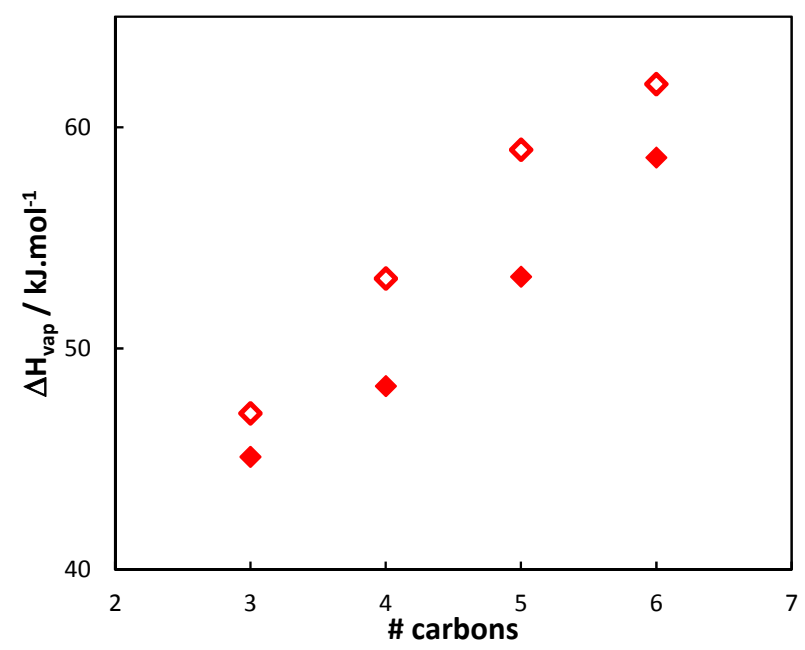

Figure 4. Molar enthalpies of vaporization of the FTOH family from experiment $(\diamond)$ and simulation $(\diamond)$.

\subsection{Liquid densities and thermal expansion coefficients}

The experimental liquid densities for 2:1, 3:1, 4:1 and 5:1 FTOH are collected in table 5 at 5 $\mathrm{K}$ intervals, in the $278 \mathrm{~K}-353 \mathrm{~K}$ temperature range, and are plotted in figure 5 . The complete table of densities can be found in the Supplementary Information. 
Table 5. Densities for $2: 1,3: 1,4: 1$ and 5:1 FTOH at $p=0.1 \mathrm{MPa}{ }^{a}$

\begin{tabular}{cc|cc|cc|cc}
\multicolumn{2}{c|}{$\mathbf{2 : 1} \mathbf{F T O H}$} & \multicolumn{2}{c|}{$\mathbf{3 : 1} \mathbf{F T O H}$} & \multicolumn{2}{c|}{$\mathbf{4 : 1} \mathbf{F T O H}$} & \multicolumn{2}{c}{$\mathbf{5 : 1} \mathbf{F T O H}$} \\
\hline $\mathbf{T} / \mathbf{K}$ & $\boldsymbol{\rho} / \mathbf{g . c m}^{-\mathbf{3}}$ & $\mathbf{T} / \mathbf{K}$ & $\boldsymbol{\rho} / \mathbf{g . c m}$ & $\mathbf{T} / \mathbf{K}$ & $\boldsymbol{\rho} / \mathbf{g . c m}^{-3}$ & $\mathbf{T} / \mathbf{K}$ & $\boldsymbol{\rho} / \mathbf{g . c m}^{-\mathbf{3}}$ \\
\hline 278.156 & 1.542779 & 278.155 & 1.633587 & 278.150 & 1.689575 & 278.151 & 1.746400 \\
283.147 & 1.533693 & 283.148 & 1.624226 & 283.149 & 1.680180 & 283.148 & 1.737071 \\
288.146 & 1.524473 & 288.147 & 1.614728 & 288.147 & 1.670671 & 288.147 & 1.727618 \\
293.146 & 1.515119 & 293.147 & 1.605106 & 293.148 & 1.661048 & 293.149 & 1.718063 \\
298.146 & 1.505629 & 298.146 & 1.595340 & 298.149 & 1.651303 & 298.148 & 1.708376 \\
303.147 & 1.495975 & 303.148 & 1.585414 & 303.148 & 1.641419 & 303.148 & 1.698566 \\
308.146 & 1.486147 & 308.146 & 1.575323 & 308.148 & 1.631401 & 308.149 & 1.688624 \\
313.145 & 1.476122 & 313.145 & 1.565049 & 313.148 & 1.621228 & 313.148 & 1.678543 \\
318.147 & 1.465891 & 318.147 & 1.554576 & 318.149 & 1.610899 & 318.148 & 1.668318 \\
323.145 & 1.455434 & 323.145 & 1.543907 & 323.148 & 1.600404 & 323.148 & 1.657947 \\
328.146 & 1.444737 & 328.146 & 1.533018 & 328.148 & 1.589737 & 328.149 & 1.647416 \\
333.144 & 1.433788 & 333.145 & 1.521908 & 333.148 & 1.578896 & 333.148 & 1.636724 \\
338.144 & 1.422580 & 338.144 & 1.510567 & 338.148 & 1.567872 & 338.148 & 1.625880 \\
343.149 & 1.411103 & 343.145 & 1.498990 & 343.148 & 1.556652 & 343.148 & 1.614863 \\
348.146 & 1.399338 & 348.145 & 1.487165 & 348.150 & 1.545153 & 348.149 & 1.603675 \\
353.145 & 1.387286 & 353.145 & 1.475083 & 353.146 & 1.533524 & &
\end{tabular}

${ }^{a}$ Standard uncertainties $u$ are $u(T)=0.01 \mathrm{~K}$ and $U(\rho)=0.2 \%$

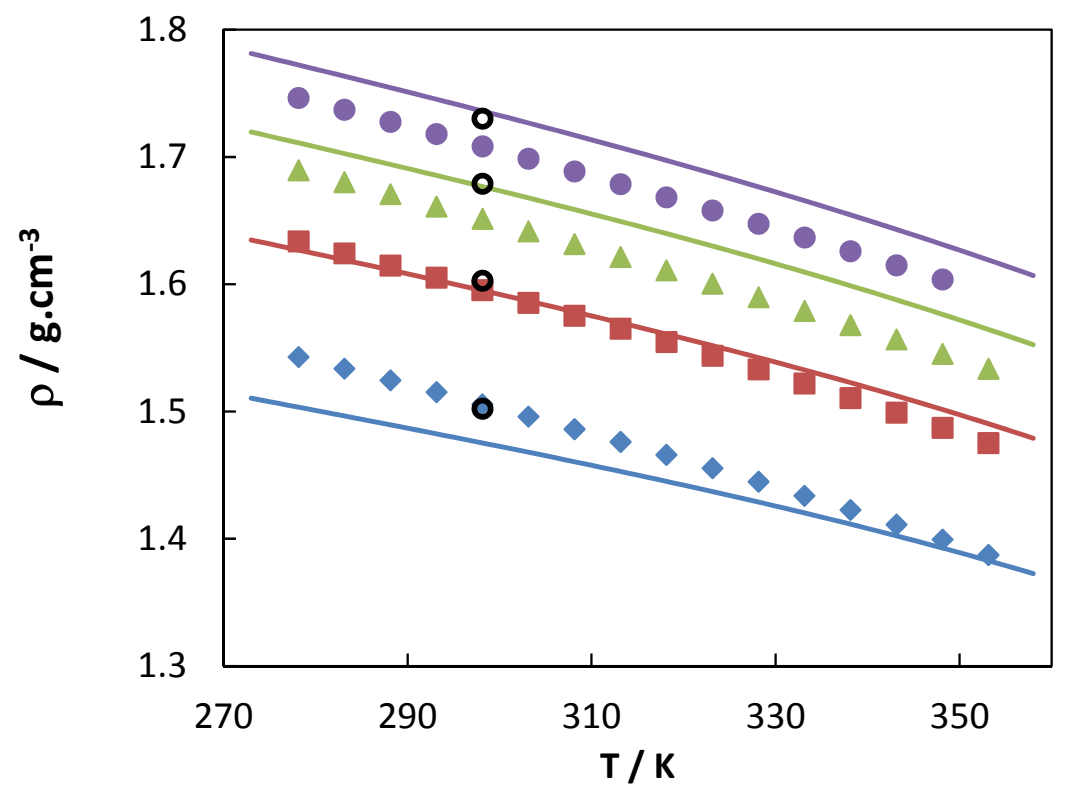

Figure 5. Experimental liquid densities and GC-SAFT-VR (lines) and simulation (0) predictions for 2:1 ( $\bullet$,blue), 3:1 ( $\boldsymbol{\bullet}$,red), 4:1 ( $\boldsymbol{\Delta}$,green) and 5:1 FTOH ( $\bullet$,purple).

Third degree polynomial equations were fitted to the experimental densities in the measured temperature range. The fitting constants are recorded in table 6 . 


$$
\rho\left(\text { g.cm } \mathrm{cm}^{-3}\right)=a_{3}(T / 100)^{3}+a_{2}(T / 100)^{2}+a_{1}(T / 100)+a_{0}
$$

Table 6. Coefficients for equation 4.

\begin{tabular}{ccccc} 
Compound & $\mathbf{a}_{\mathbf{3} .10^{2}}$ & $\mathbf{a}_{\mathbf{2}} \cdot \mathbf{1 0}^{\mathbf{2}}$ & $\mathbf{a}_{\mathbf{1}} \cdot \mathbf{1 0}^{\mathbf{1}}$ & $\mathbf{a}_{\mathbf{0}}$ \\
\hline 2:1 FTOH & -1.913547 & 0.1385471 & -5.073642 & 2.293902 \\
3:1 FTOH & -1.419722 & 9.531180 & -3.867149 & 2.277346 \\
4:1 FTOH & -1.122842 & 7.409053 & -3.386162 & 2.299856 \\
5:1 FTOH & -0.6976345 & 3.693059 & -2.291108 & 2.248078
\end{tabular}

The liquid densities obtained from molecular dynamics simulations at $298.15 \mathrm{~K}$ are compared with the experimental data in table 7 and in figure 5 . The simulations are able to predict the density with deviations of less than $2 \%$ to the experimental results, which is well within the deviations obtained in the original OPLS-AA work. [8] This result, together with the fairly good estimates obtained for the vaporization enthalpies, validates the molecular model used in the simulations.

The GC-SAFT-VR predictions for the liquid density of the fluoroalcohols are also compared with the experimental data in figure 5. These theoretical predictions agree with the experimental results with maximum deviations of $2.6 \%$, and can be considered very good given that the parameters were not fitted to experimental data for FTOH molecules. While better agreement could be achieved if specific binary interaction parameters were adjusted for each substance, this would severely limit the predictive ability of the approach which is one of the advantages of a group-contribution based equation. Literature density data by Rochester et al.[26] and Denda et al.[27] at 298.15 K was available for 2:1 FTOH, with deviations of $3.6 \%$ and $2.3 \%$. This discrepancy is likely due to differences in the purity of the samples, namely water content.

Table 7. Experimental and simulated liquid densities, and thermal expansion coefficients at $298.15 \mathrm{~K}$.

\begin{tabular}{|c|c|c|c|}
\hline Substance & $\rho_{\exp }\left(\right.$ g.cm $\left.{ }^{-3}\right)$ & $\rho_{\text {sim }}\left(\right.$ g.cm $\left.{ }^{-3}\right)$ & $\alpha\left(\mathrm{K}^{-1}\right) \cdot 10^{3}$ \\
\hline 2:1 FTOH & 1.505624 & 1.502 & 1.27 \\
\hline 3:1 FTOH & 1.595328 & 1.603 & 1.23 \\
\hline $4: 1 \mathrm{FTOH}$ & 1.651294 & 1.679 & 1.19 \\
\hline 5:1 FTOH & 1.708357 & 1.730 & 1.14 \\
\hline
\end{tabular}

Finally, in figure 6 the thermal expansion coefficients, $\alpha$, obtained from the temperature dependence of the experimental liquid densities by differentiation of equation 14, are plotted as a function of chain length. As could be expected, $\alpha$ decreases with the length of the FTOH molecule. 


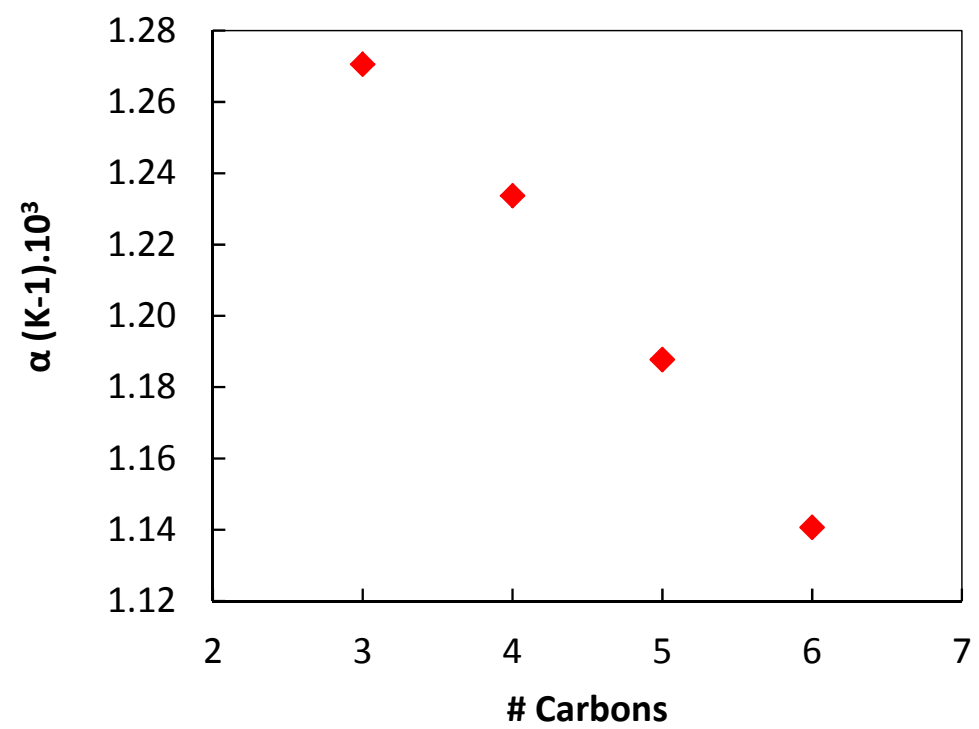

Figure 6. Thermal expansion coefficient at $298.15 \mathrm{~K}$ vs chain length.

\subsection{Molecular simulations}

The average number of hydrogen bonds per alcohol molecule $\left(n_{\mathrm{HB}}\right)$ was obtained from the molecular dynamics trajectories and is represented in figure 7 . We have also included in the figure similar results for trifluoroethanol and a number of hydrogenated alcohols from the literature, obtained with various force fields (OPLS-AA, OPLS-UA and TraPPE models). For the latter it is not possible to discern a clear tendency for the average number of hydrogen bonds, and the results do not seem to be sensitive to different molecular models or to the slightly different criteria used to identify and count hydrogen bonds. The fluorinated alcohols, however, seem to present an increasing number of hydrogen bonds as the chain grows, but always remaining below the values for their hydrogenated counterparts. This lower number of hydrogen bonds in the liquid phase may contribute to the lower enthalpies of vaporization observed. 


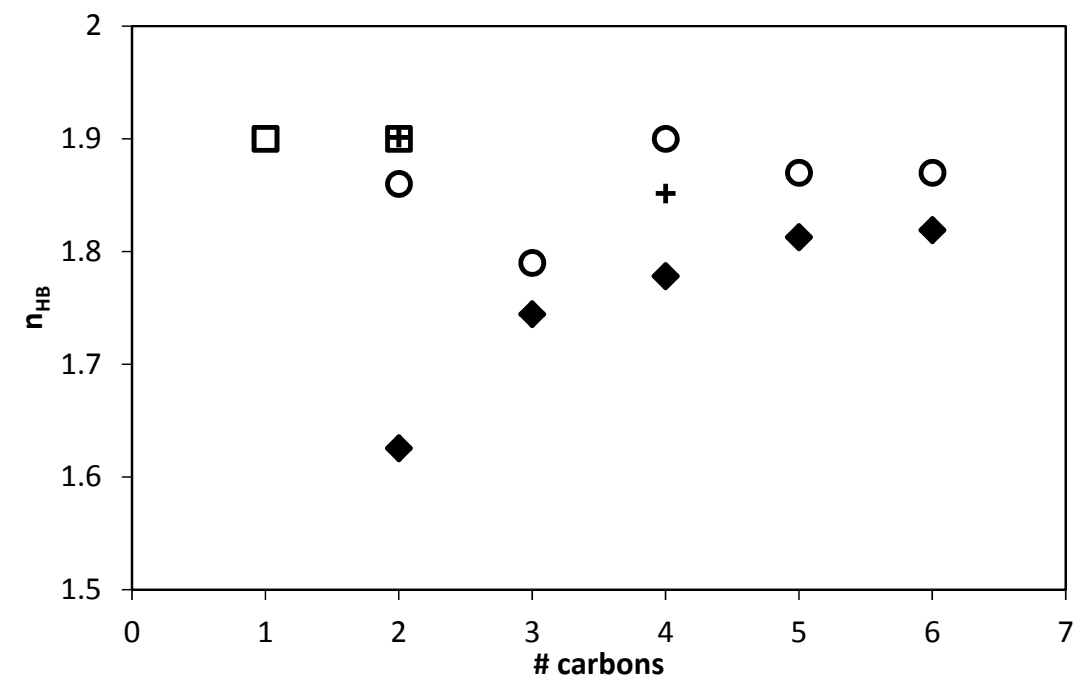

Figure 7. Average number of hydrogen bonds per molecule vs chain length, obtained by molecular dynamics. $(\diamond)$ FTOH this work (OPLS-AA model); (+) Ethanol and butanol from Ref.[28] (OPLS-AA model) ; ( $\square$ ) Methanol and ethanol from Ref.[29] (OPLS-UA model); (O) Ethanol to Hexanol from Ref.[30] (TraPPE model).

A conformational analysis of the fluoroalcohols was also obtained from the simulations for the liquid and the gas phases. The dihedral distribution function (DDF) is shown in figure 8 in a symmetric representation where $180^{\circ}$ corresponds to the fully trans conformation, (in which the hydroxyl hydrogen points away from the molecule backbone) and $0^{\circ}$ is the opposite configuration (in which the hydroxyl hydrogen points towards the second carbon atom). As can be seen, the DDF are similar for all the fluoroalcohols. In the gas phase a single peak is observed, centered around $70^{\circ}$, which corresponds to a gauche conformer. In the liquid phase, this peak shifts to higher angles, $\sim 90^{\circ}$, and there is now a significant probability of finding transoid conformers, with dihedral angles close to $180^{\circ}$. This shift is caused by hydrogen bonding between different molecules, which are favored by higher dihedral angles. In the gas phase, however, intramolecular electrostatic interactions between the hydroxyl hydrogen and the negatively charged fluorine atoms increase the number of gauche conformations and lower the energy of the molecule in the vapor. This intramolecular interaction is not possible in the hydrogenated alcohols, and is likely to contribute to the lower enthalpy of vaporization observed for the fluorinated alcohols. 


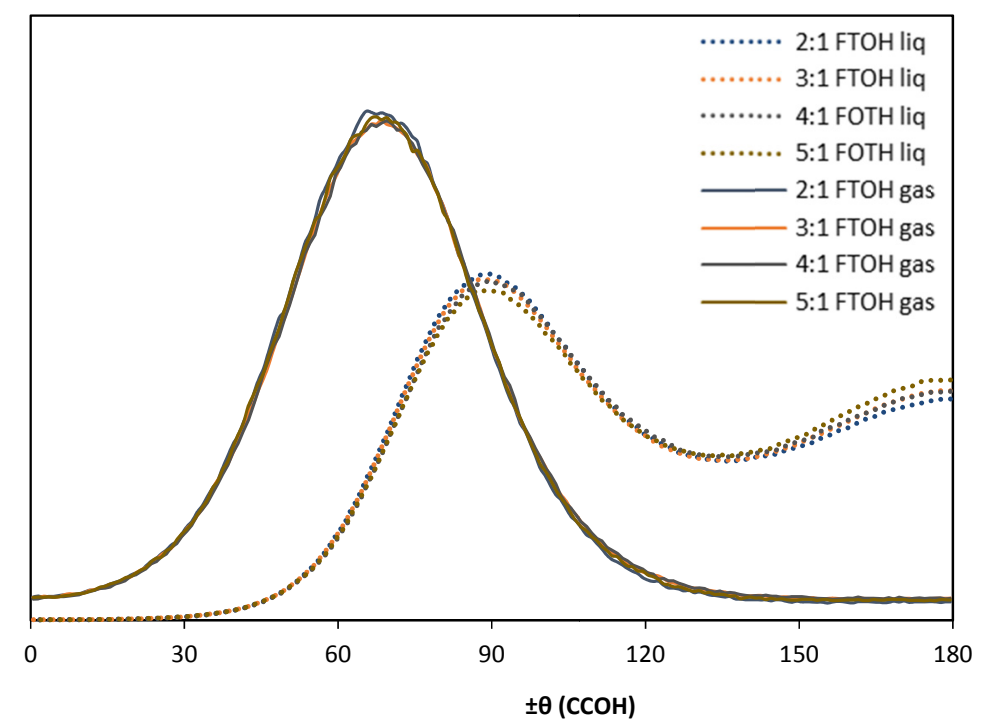

Figure 8. Dihedral distribution function around the C-O bond.

\section{Conclusions}

The vapor pressure of $1 \mathrm{H}, 1 \mathrm{H}$-perfluoroalcohols $(\mathrm{CF} 3(\mathrm{CF} 2) \mathrm{n}(\mathrm{CH} 2) \mathrm{OH}, \mathrm{n}=1,2,3,4)$ was measured in the temperature range between $278 \mathrm{~K}$ and $328 \mathrm{~K}$, and the liquid density of the same compounds was measured between $278 \mathrm{~K}$ and $353 \mathrm{~K}$. Enthalpies of vaporization were calculated from the experimental data and a linear dependency with chain length was observed for the odd fluorotelomer alcohols family, much like the dependency for alkanols and with roughly the same slope, while the absolute values are comparatively lower.

The results were modelled with the GC-SAFT-VR EoS, using a purely predictive approach, and very good agreement for the vapor pressures and enthalpies of vaporization was obtained. The liquid density curves were also predicted with a good degree of agreement.

Molecular dynamics simulations were performed in order to obtain molecular level information about the behavior of the studied substances. The liquid densities and the enthalpies of vaporization obtained from the simulations showed good agreement with the experimental results, validating the force field used. Further analysis of the simulation results showed that the number of hydrogen bonds per molecule of fluorinated alcohol increases with chain length, but remains lower than that reported in the literature for hydrogenated nalcohols; this factor surely contributes to the lower enthalpy of vaporization observed for the fluorinated alcohols. The dihedral distribution function (ddf) around the rotation of the C-O bond obtained from the simulation data shows that the conformational distribution of the FTOH is significantly different in the gas and in the liquid phases. In the gas phase the hydroxyl hydrogen of the fluoroalcohols is predominantly oriented towards the fluorocarbon chain, in gauche-like conformations centered at $70^{\circ}$. In the liquid, the distribution becomes broader, the gauche peak is now centered at $90^{\circ}$ and a significant fraction of molecules display a transoid conformation. The higher proportion of gauche conformers in the gas phase, stabilized by the intramolecular electrostatic interaction between the fluorine atoms 
and the hydroxyl hydrogen, also contributes to the comparatively lower enthalpy of vaporization of the FTOH.

\section{Acknowledgements}

P.M. acknowledges funding from Fundac ão para a Cie ncia e Tecnologia, in the form of Grant SFRH/BPD/81748/2011. E.J.M.F. and P.M. acknowledge support from Fundac ão para a Cie ncia e a Tecnologia through Grant Pest-OE/QUI/UI0100/2013. LFGM acknowledges funding from FCT through the grant PEst-OE/QUI/UI0619/2011. JDH, VMTM and CMC gratefully acknowledge financial support from the National Science Foundation under Grant CBET-1067642 and the U.S. Department of Energy (DOE), Office of Basic Energy Sciences, Geoscience Research Program, through Grant No. ERKCC72 of Oak Ridge National Laboratory, which is managed for DOE by UT Battelle, LLC under Contract No. DE-AC05- 00OR22725. JDH also acknowledges support from the U.S. Department of Education for a Graduate Assistance in Areas of National Need (GAANN) Fellowship under grant number P200A090323.

${ }^{1}$ Fluorinated Surfactants, E. Kissa, Marcel Dekker, NY, 1993.

${ }^{2}$ L.A.M. Pereira, L.F.G. Martins, J.R. Ascenso, P. Morgado, J.P.P. Ramalho, E.J.M. Filipe, Diffusion Coefficients of Fluorinated Surfactants in Water: Experimental Results and Prediction by Computer Simulation, J. Chem. Eng. Data 59 (2014) 3151-3159.

${ }^{3}$ P. Duarte, M. Silva, D. Rodrigues, P. Morgado, L.F.G. Martins, E.J.M. Filipe, Liquid Mixtures Involving Hydrogenated and Fluorinated Chains: ( $p, \rho, T, x)$ Surface of (Ethanol + 2,2,2-Trifluoroethanol), Experimental and Simulation., J. Phys. Chem. B 117 (2013) 9709-9717.

${ }^{4}$ G.C. Benson, H.D. Pflug, Molar excess volumes of binary systems of normal alcohols at 25.deg., J. Chem. Eng. Data 15 (1970) 382-386.

${ }^{5}$ E. Matteoli, L. Lepori, Determination of the excess enthalpy of binary mixtures from the measurements of the heat of solution of the components: application to the perfluorohexane + hexane mixture, Fluid Phase Equilib. 174 (2000) 115-131.

${ }^{6}$ J.C.S. Costa, M. Fulem, B. Schröder, J.A.P. Coutinho, M.J.S. Monte, L.M.N.B.F. Santos, Evidence of an odd-even effect on the thermodynamic parameters of odd fluorotelomer alcohols, J. Chem. Thermodyn. 54 (2012) 171-178.

${ }^{7}$ P. Morgado, G. Das, C. McCabe, E.J.M. Filipe, Vapor Pressure of Perfluoroalkylalkanes: The Role of the Dipole, J. Phys. Chem. B 119 (2015) 1623-1632.

${ }^{8}$ W.L. Jorgensen, D.S. Maxwell, J. Tirado-Rives, Development and Testing of the OPLS AllAtom Force Field on Conformational Energetics and Properties of Organic Liquids, J. Am. Chem. Soc. 118 (1996) 11225-11236.

${ }^{9}$ E.K. Watkins, W.L. Jorgensen, Perfluoroalkanes: Conformational Analysis and LiquidState Properties from ab initio and Monte Carlo Calculations, J. Phys. Chem. A 105 (2001) 4118-4125.

${ }^{10}$ E.M. Duffy, Ph.D. Thesis, Yale University, New Haven, CT, 1994.

${ }^{11}$ R. Chitra, P.E. Smith, A comparison of the properties of 2,2,2-trifluoroethanol and 2,2,2trifluoroethanol/water mixtures using different force fields, J. Chem. Phys. 115 (2001) 5521-5530. 
${ }^{12}$ A.A.H. Pádua, Torsion Energy Profiles and Force Fields Derived from Ab Initio Calculations for Simulations of Hydrocarbon-Fluorocarbon Diblocks and Perfluoroalkylbromides, J. Phys. Chem. A 106 (2002) 10116-10123.

${ }^{13}$ W. Smith, T.R. Forester, I.T. Todorov, The DL_POLY Classic User Manual; Daresbury Laboratory, U.K., 2012.

${ }^{14}$ M. Brehm, B. Kirchner, TRAVIS - A free Analyzer and Visualizer for Monte Carlo and Molecular Dynamics Trajectories, J. Chem. Inf. Model. 51 (2011) 2007-2023.

${ }^{15}$ A. Gil-Villegas, A. Galindo, P.J. Whitehead, S.J. Mills, G. Jackson, A.N. Burgess, Statistical associating fluid theory for chain molecules with attractive potentials of variable range, J. Chem. Phys. 106 (1997) 4168-4186.

${ }^{16}$ Y. Peng, K.D. Goff, M.C. Dos Ramos, C. McCabe, Developing a predictive groupcontribution-based SAFT-VR equation of state, Fluid Phase Equilib. 277 (2009) 131-144.

${ }^{17}$ C. McCabe, A. Gil-Villegas, G. Jackson and F. del Rio, The thermodynamics of heteronuclear molecules formed from bonded square-well (BSW) segments using the SAFT-VR approach, Mol. Phys. 97 (1999) 551-558.

${ }^{18}$ Y. Peng, H. G. Zhao and C. McCabe, On the thermodynamics of diblock chain fluids from simulation and heteronuclear statistical associating fluid theory for potentials of variable range, Mol. Phys. 104 (2006) 571-586.

${ }^{19}$ C. McCabe, G. Jackson, SAFT-VR modelling of the phase equilibrium of long-chain nalkanes, Phys. Chem. Chem. Phys. 1 (1999) 2057-2064.

${ }^{20}$ P.J. Leonard, D. Henderson, J.A. Barker, Perturbation Theory and Liquid Mixtures, Trans. Faraday Soc. 66 (1970) 2439-2452.

${ }^{21}$ Jessica D. Haley, M. C. d. R., and Clare McCabe In Preparation 2015.

${ }^{22}$ M.C. dos Ramos, J.D. Haley, J.R. Westwood, C. McCabe, Extending the GC-SAFT-VR approach to associating functional groups: Alcohols, aldehydes, amines and carboxylic acids, Fluid Phase Equilib. 306 (2011) 97-111.

${ }^{23}$ A.C. Meeks, I.J. Goldfarb, Vapor Pressure of Fluoroalcohols, J. Chem. Eng. Data. 12 (1967) 196.

${ }^{24}$ P.J. Linstrom, W.G. Mallard, Eds., NIST Chemistry WebBook, NIST Standard Reference Database Number 69, National Institute of Standards and Technology, Gaithersburg MD, 20899, http://webbook.nist.gov, (retrieved July 29, 2015).

${ }^{25}$ R.A. Cormanich, R. Rittner, M.P. Freitas, M. Bühl, The seeming lack of CF $\cdots H O$ intramolecular hydrogen bonds in linear aliphatic fluoroalcohols in solution, Phys. Chem. Chem. Phys. 16 (2014) 19212-19217.

${ }^{26}$ Rochester, C. H., Symonds, J. R., Thermodynamic studies of fluoroalcohols. Part 1.Vapour pressures and enthalpies of vaporization, J. Chem. Soc., Faraday Transactions 1, 69 (1973) 1267.

${ }^{27}$ M. Denda, H. Touhara, K. Nakanishi, Excess molar enthalpies for (water + a fluoroalkanol), J. Chem. Thermodyn. 19 (1987) 539.

${ }^{28}$ P. Morgado, A. R. Garcia, L. M. Ilharco, J. Marcos, M. Anastácio, L. F. G. Martins and E. J. M. Filipe, Liquid Mixtures Involving Hydrogenated and Fluorinated Alcohols: Thermodynamics, Spectroscopy and Simulation, submitted to J. Phys. Chem. B.

${ }^{29}$ J.A. Padró, L. Saiz, E. Guàrdia, Hydrogen bonding in liquid alcohols: A computer simulation study, J. Mol. Struct. 416 (1997) 243-248.

${ }^{30}$ M. Tomšič, A. Jamnik, G. Fritz-Popovski, O. Glatter, L. Vlček, Structural properties of pure simple alcohols from ethanol, propanol, butanol, pentanol, to hexanol: Comparing Monte Carlo simulations with experimental SAXS data, J. Phys. Chem. B. 111 (2007) $1738-1751$. 